\title{
Maximal Rate of the Left Ventricular Pressure Fall (Peak Negative dP/dt) in Early Stage of Myocardial Ischemia Following Experimental Coronary Occlusion
}

\author{
Tan Watanabe, M.D., Fujio Shintani, M.D., Long-Tai Fu, M.D., \\ and Kazuzo Kato, M.D.
}

\begin{abstract}
SUMMARY
Short-term, repeated myocardial ischemia was produced in 20 opened-chest anesthetized dogs by left anterior descending (LAD) branch occlusion. Left ventricular pressure (LVP) and its $\mathrm{dP} / \mathrm{dt}$ were recorded by Satham SF-1 transducer, and isometric local force (F) was measured by a Walton-Brodie strain gauge arch sewn onto LV free wall. In all experiments, within a few beats after LAD occlusion, peak negative $\mathrm{dP} / \mathrm{dt}$ started to diminish its amplitude, reaching a nadir after $20-30 \mathrm{sec}(63 \pm 5 \%$ of control, $9 \mathrm{dogs}$ ). It recovered to $83 \pm 3 \%$ of control level in $45 \mathrm{sec}$ and remained essentially unchanged thereafter. This change preceded those of LVP and max $\mathrm{dP} / \mathrm{dt}$, which showed only minor falls, In 6 dogs, changes by occluding root of LAD were compared with those by its branch occlusion. The greater the extent of ischemia, the more marked and prolonged were the changes. While $\mathrm{F}$ in the ischemic center (Fc) rapidly lost its contractile activity following LAD occlusion, $\mathrm{F}$ in marginal zone between infarcted and non-infarcted region (Fm) showed variable extent and different time-course of its diminution. Thus, it was concluded that the change in peak negative $\mathrm{dP} / \mathrm{dt}$ following LAD occlusion was one of the sensitive signs of early ischemic changes, and was attributed at least in part to characteristic changes in contractile activity of myocardium surrounding the ischemic injury.
\end{abstract}

Additional Indixing Words :

Size of ischemic injury Marginal zone Local force

W

HILE the factors influencing the rate of pressure development in the left ventricle (positive $\mathrm{dP} / \mathrm{dt}$ ) have been extensively studied, ${ }^{1 /-4)}$ little attention has been paid to the process of the ventricular relaxation. Recently, it was reported that maximal rate of the left ventricular pressure fall (negative $\mathrm{dP} / \mathrm{dt}$ ) was related both to the intrinsic contractility of the heart and to the volume of the heart at which relaxation commenced. ${ }^{5}$ Although the occurrence of a defect in myocardial relaxation during ischemia has been suggested

From the Cardiovascular Institute (Shinken), 8-1-22 Akasaka, Minato-ku, Tokyo 107, Japan.

Received for publication December 11, 1974. 
by a recent study, ${ }^{6}$ ' precise investigations on negative $\mathrm{dP} / \mathrm{dt}$ in the ischemic heart are yet meager. We have noticed that a characteristic change in peak negative $\mathrm{d} \mathbf{P} / \mathrm{dt}$ during isometric relaxation phase was produced with little changes in positive $\mathrm{dP} / \mathrm{dt}$ by a short-term coronary occlusion in experimental dogs. ${ }^{7,8)}$ Then an attempt was made to observe the natural course of this change in peak negative $\mathrm{dP} / \mathrm{dt}$ following an acute coronary occlusion and to better define their determinants with the aid of registration of left ventricular local forces. The purpose of this paper is to present these results, which facilitate precise analysis of the left ventricular relaxation process and better understanding of the changes in diastolic compliance during acute myocardial ischemia.

\section{Materials and Methods}

Studies were carried out in 20 mongrel dogs weighing between 9 to $15 \mathrm{Kg}$ and anesthetized with $25 \mathrm{mg} / \mathrm{Kg}$ of sodium pentobarbital. Respiration was maintained with a Harvard respirator, and heart was exposed through a left thoracotomy and suspended in a pericardial cradle. One of the primary branches of the left anterior descending (LAD) coronary artery was dissected free for the repeated occlusions. In 8 experiments, the root of LAD was dissected as well.

Left ventricular pressure (LVP) was measured by a Statham F-l cathetertip transducer, inserted into $\mathrm{LV}$ cavity via the left carotid artery. The $\mathrm{LV} \mathrm{dP} / \mathrm{dt}$ was registered with use of a Hewlett-Packard Model 8814A derivative computer. Aortic pressure was measured through a metal cannula inserted via the right carotid artery, connected to a Statham P23Db transducer. In 3 dogs, aortic flow was registered by applying a probe around the root of the aorta, connecting it to a Statham M-4001 electromagnetic flowmeter. A Walton-Brodie strain gauge arch was sutured onto LV free wall in 13 experimental animals. In some, feet of the arch were positioned so as to be located in the expected center of ischemic zone following LAD occlusion. In the other, location of the arch was selected to be positioned at around the marginal zone between infarcted and non-infarcted region. The LV surface electrogram (ECG) was recorded in all experiments with an electrode positioned within the ischemic lesion following LAD occlusion. All variables were recorded on a Clevite-Brush Model 260 multichannel oscillographic recorder.

\section{Results}

\section{Time Course of Peak Negative dP/dt Following LAD Occlusion}

Representative changes in the variables before and after LAD branch occlusion are shown in Fig. 1. A slight fall in LVP and elevation in LVEDP were noticed after $20 \mathrm{sec}$ following the occlusion. Positive $\mathrm{dP} / \mathrm{dt}$ did not change until $25 \mathrm{sec}$ after occlusion, when it began to fall slightly. In contrast, peak negative $\mathrm{dP} / \mathrm{dt}$ began to decrease its amplitude immediately after the 


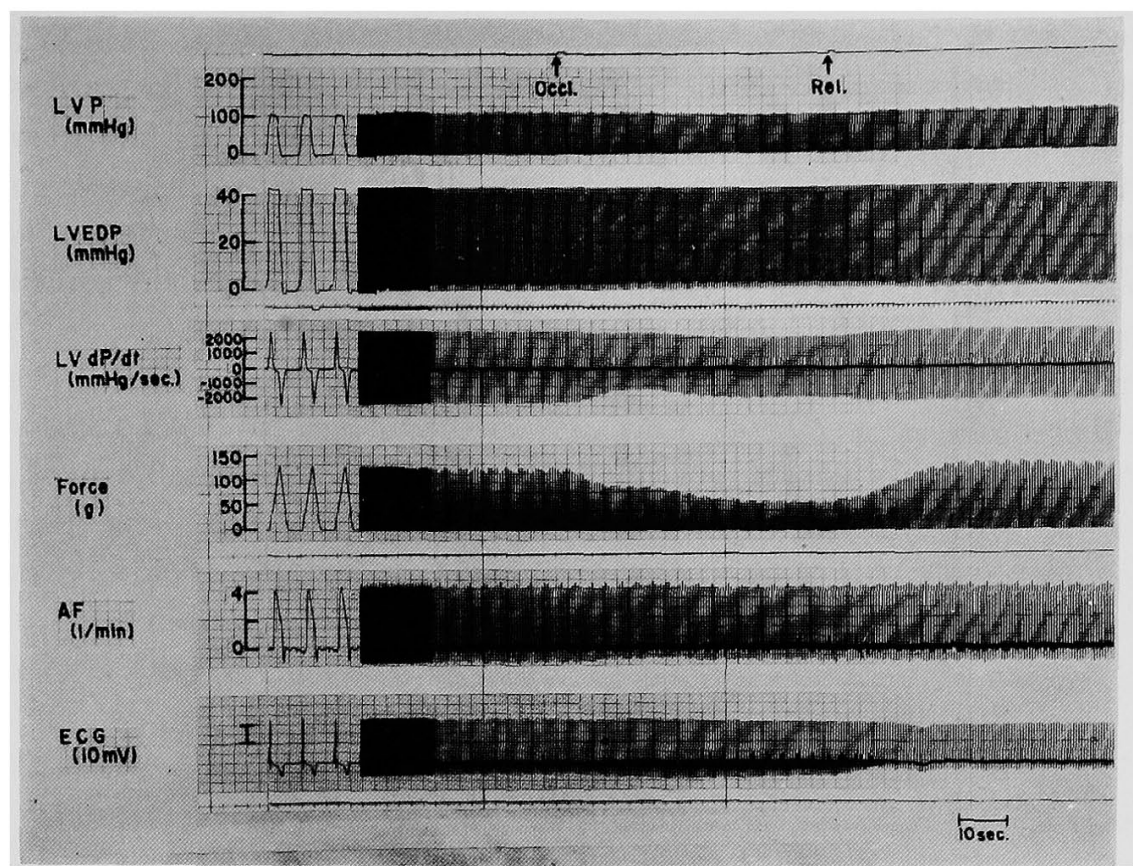

Fig. 1. Typical recording obtained before and during LAD branch occlusion. $\quad L V P=$ left ventricular pressure; $L V E D P=$ left ventricular enddiastolic pressure; $\mathrm{LV} \mathrm{dP} / \mathrm{dt}=$ rate of change of $\mathrm{LVP}$; Force=isometric local force; $\mathrm{AF}=$ aortic flow rate; $\mathrm{ECG}=$ electrocardiogram obtained from the center of ischemia.

occlusion, reaching a nadir at about 10 to $15 \mathrm{sec}$ (identical to 20 to 30 th heart beat). Then, it started to recover toward its control level. Recovery to about $80 \%$ of the control was achieved for $20 \mathrm{sec}$, but no more change was found throughout the occlusion. Local force recorded from the center zone of ischemia started to diminish immediately after the occlusion and continued to diminish progressively throughout the period of occlusion. No marked changes were noticed in peak velocity in the aortic flow. ECG recorded from the center zone of ischemia showed an augmentation of negative T-wave following occlusion before S-T segment elevation.

Fig. 2 depicts the average changes in peak negative $\mathrm{dP} / \mathrm{dt}$ following LAD branch occlusion in 9 dogs. Changes in amplitude of peak negative $\mathrm{dP} / \mathrm{dt}$ measured at every $5 \mathrm{sec}$ are expressed as percent of control value on vertical axis. An initial rapid reduction to $62 \pm 5 \%$ (mean $\pm \mathrm{SEM}$ ) at $15 \mathrm{sec}$ after occlusion was followed by a gradual recovery to about $82 \%$ of the control level.

A record at a fast papar speed is shown in Fig. 3 in order to demonstrate the differences in contraction patterns obtained before LAD occlusion, at the 


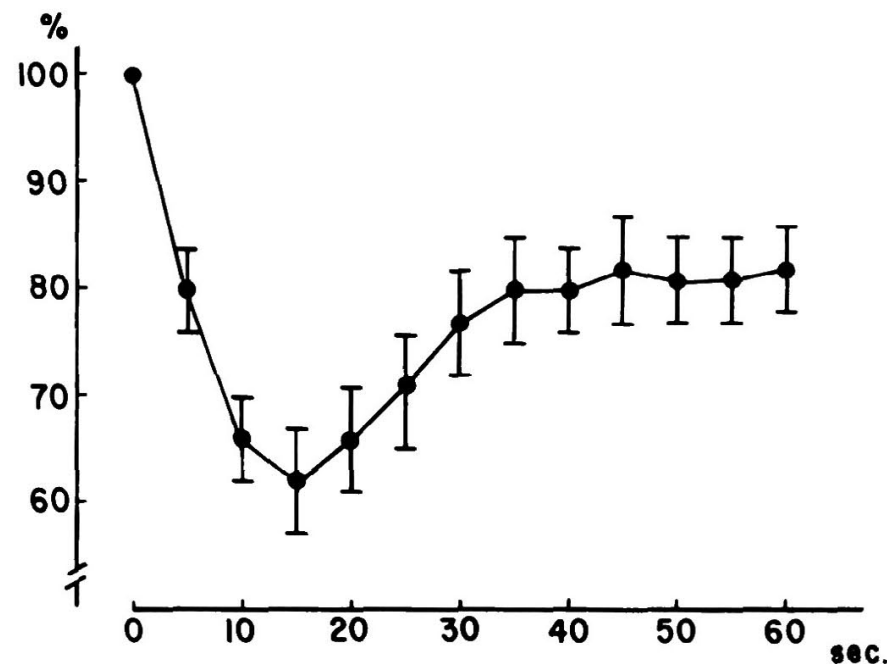

Fig. 2. Average changes in peak negative $\mathrm{dP} / \mathrm{dt}$ following $60 \mathrm{sec} \mathrm{LAD}$ branch occlusion in 9 experiments. Circle and bar represent mean value and SEM, respectively.

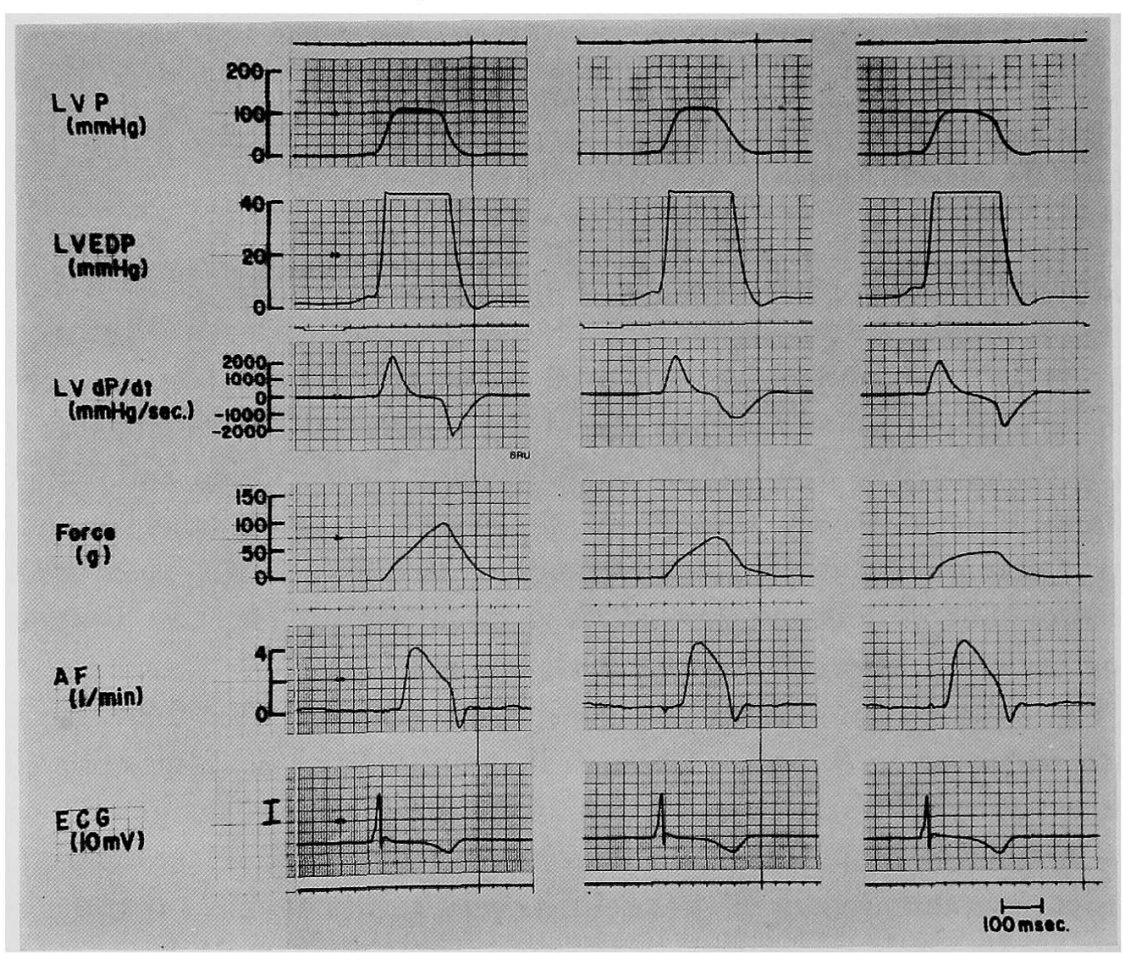

Fig. 3. Typical recordings at a fast paper speed obtained before LAD occlusion (left), at the time of nadir of peak negative $\mathrm{dP} / \mathrm{dt}$ (middle), and immediately prior to release of the occlusion (right). 
nadir of peak negative $\mathrm{dP} / \mathrm{dt}$, and prior to release of occlusion. LVP pattern at the time of the nadir of peak negative $\mathrm{dP} / \mathrm{dt}$ is characterized by a short duration and its earlier fall of peak pressure with decreased rate of its fall during isometric relaxation phase, as compared with that of control. No essential change in duration of total contraction is observed. In other words, insufficient maintenance of late systolic pressure is noted. These changes in LVP pattern are clearly recovered prior to release of occlusion, although peak LVP decreased slightly. Peak negative $\mathrm{dP} / \mathrm{dt}$ shows changes reflecting the altered LVP patterns above mentioned. That is, decreased amplitude with longer duration of negative $\mathrm{dP} / \mathrm{dt}$ in the middle panel is noted, while the right panel shows only slight decrease in the amplitude. Ejection time measured from the aortic flow is markedly shortened at the time of the nadir of peak negative $\mathrm{dP} / \mathrm{dt}$. Along with augmentation of negativity of $\mathrm{T}$-wave in ECG, a Q-T prolongation is noticed in both phases after the occlusion.

\section{Relation of the Size of Ischemic Area to Changes in Peak Negative dP/dt}

The changes in peak negative $\mathrm{dP} / \mathrm{dt}$ following occlusion of the root of LAD is compared with that following its major branch occlusion in Fig. 4. Mean values and SEM obtained at every fifth heart beat in 6 experiments are shown. A significant and prolonged changes in peak negative $\mathrm{dP} / \mathrm{dt}$ are associated with greater ischemic area following occlusion of root of LAD (solid line), comparing with those following the branch occlusion (broken line). The amplitude of peak negative $\mathrm{dP} / \mathrm{dt}$ diminished more rapidly, reaching a

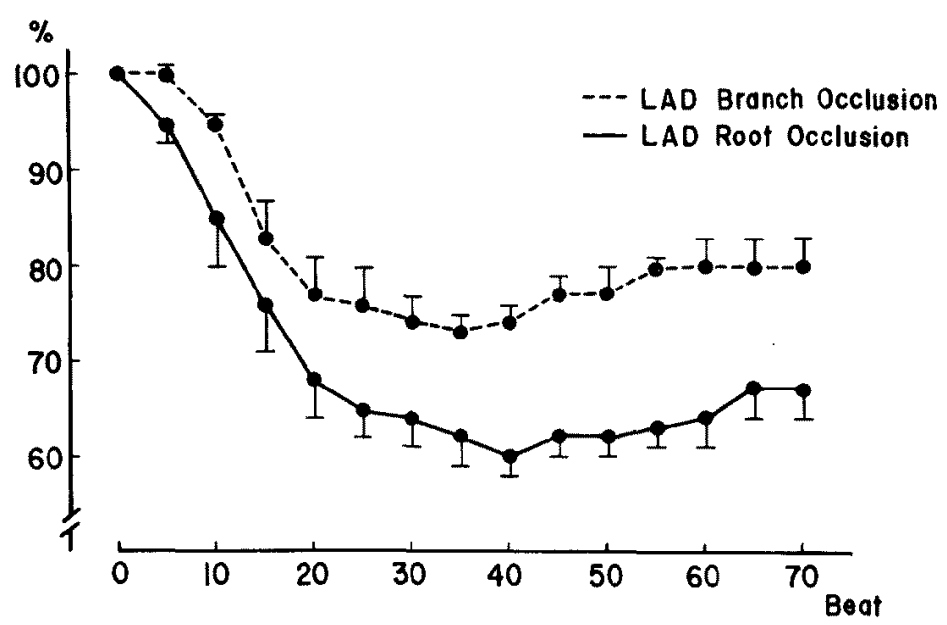

Fig. 4. Comparison of the changes in peak negative $\mathrm{dP} / \mathrm{dt}$ following LAD-root occlusion (solid line) with that following its branch occlusion (broken line) in 6 experiments. 
more depressed and delayed nadir. The difference in extent of the decrease from the occlusion of branch of LAD is statistically significant at most of heart beats of identical number (Student's paired t-test).

Changes in Local Force: One of the Determinants of Peak Negative dP/dt following LAD Occlusion

As shown in Figs. 1 and 3, an immediate decline was brought about in the amplitude of local force (Fc) at the central zone of ischemic injury by LAD occlusion. The force continued decreasing throughout the entire period of occlusion unlike the peak negative $\mathrm{dP} / \mathrm{dt}$, which showed marked recovery after initial decrease. On the other hand, the marginal zone between infarcted and non-infarcted region exhibited diminution in local force ( $\mathrm{Fm}$ ) of variable extent and of different time-course according to the location of force gauge. Fig. 5-A shows a record in an experiment, in which a small but discernible recovery of Fm follows a marked decline occurring in $30 \mathrm{sec}$ after the occlusion. In another experiment illustrated in Fig. 5-B, rather small initial decrease in Fm is followed by significant recovery and augmentation to a level exceeding the control.

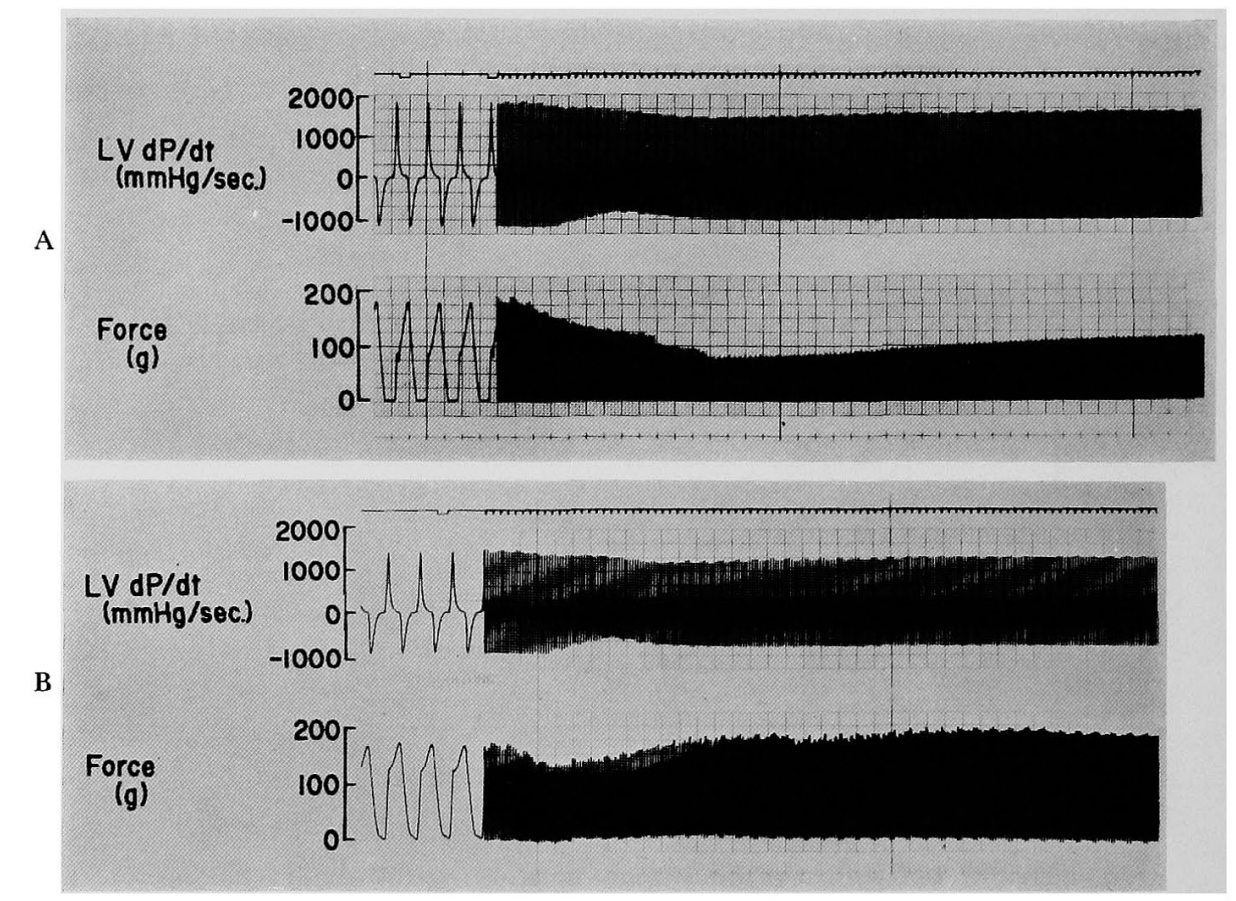

Fig. 5-A and B. Variable extent and different course of local forces obtained from marginal portion of ischemic lesion following LAD occlusion. 


\section{DisGussion}

Recently, Gohn et al reported that peak negative $\mathrm{dP} / \mathrm{dt}$ was related both to the intrinsic contractility of the heart and to the volume of the ventricle when relaxation commenced. ${ }^{5}$ ) They also noticed that inotropic stimulation might lead to bidirectional effects on negative $\mathrm{dP} / \mathrm{dt}$. The first, the direct effect is to increase negative $\mathrm{dP} / \mathrm{dt}$, whereas the second is that endsystolic volume declines, reducing peak negative $\mathrm{dP} / \mathrm{dt}$ at the same time. Then the net results might be a relatively constant negative $\mathrm{dP} / \mathrm{dt}$. And also, when negative inotropic intervention is applied, changes in negative $\mathrm{dP} / \mathrm{dt}$ might similarly be little by opposing mechanisms as above mentioned.

We confirmed in the present study that peak negative $\mathrm{d} \mathbf{P} / \mathrm{dt}$ showed a marked and characteristic changes in the early stage of coronary branch occlusion, when posititve $\mathrm{dP} / \mathrm{dt}$ was little affected. Thus, the changes in peak negative $\mathrm{dP} / \mathrm{dt}$ is one of the sensitive hemodynamic signs of early ischemic injury following coronary occlusion. McLaurin et al more recently observed that atrial pacing-induced tachycardia produced impaired LV relaxation in patients with coronary heart disease, but not in those with normal LV function.9) The finding that temporarily aggravated myocardial ischemia is associated with depressed rate of LV relaxation, is principally in agreement with those of the present study. Characteristic evidence of the pressure-volume relationship during $\mathrm{LV}$ diastole, namely, diastolic compliance has been elucidated with recent studies, especially in the presence of acute or chronic ischemic injury. ${ }^{10)-12}$ Accordingly, these findings suggest that an abnormal relaxation of the heart may be partly responsible for the apparent decrease in $\mathrm{LV}$ diastolic compliance in the presence of myocardial ischemia.

Different from several data obtained from isolated cardiac muscle, ${ }^{13)-15}$ ) no significant change was noticed in total duration of $\mathrm{LV}$ contraction after LAD occlusion, determined from LV contraction pattern (Fig. 3). In contrast, ejection period was significantly shortened in the present experiments, resulting from insufficient maintenance of peak or near-peak LVP. At this time, isometric contraction phase was slightly, and isometric relaxation phase was markedly prolonged, resulting in a marked decline in peak negative $\mathrm{dP} / \mathrm{dt}$. Thus, it was demonstrated that in in-situ heart, reduction of peak negative $\mathrm{dP} / \mathrm{dt}$ following coronary occlusion is rather attributable to prolonged isometric relaxation phase than changes in total duration of $\mathrm{LV}$ contraction.

As compared in Fig. 4, the greater the extent of ischemia, the more marked and prolonged was the diminution of amplitude in peak negative $\mathrm{dP} / \mathrm{dt}$. Since rapid loss of myocardial contractile activity in the ischemic zone is responsible for the impaired maintenance of late systolic LVP and diminished peak nega- 
tive $\mathrm{dP} / \mathrm{dt}$, it is natural to postulate that the amount of non-functioning myocardial mass relates inversely to the amplitude of peak negative $\mathrm{dP} / \mathrm{dt}$. Extent and severity of the ischemic injury, thus, significantly influence the alteration in the process of relaxation, as well as that of contraction.

Of most interest was the fact that in all the experiments, decreased peak negative $\mathrm{dP} / \mathrm{dt}$ tended to recover toward its control level even before release of the occlusion. In their experimental coronary occlusion, Sedeck and Lewartowski observed the fact that ejection time fell within $30 \mathrm{sec}$ and showed a tendency to increase toward control value. ${ }^{16)}$ They felt difficulties in explaining the biphasic changes in ejection time. Time-course of the changes in ejection time and peak negative $\mathrm{dP} / \mathrm{dt}$ seems to resemble each other, and mechanism of these phenomena can be at least partly explained by the timecourse of change in Fm. Contrary to Fc, which continued decreasing throughout the occlusion, contractile activity in marginal zone showed variable extent of recovery after the initial decrease without release of the occlusion. In some animals, significantly depressed activity was recovered in small extent, while in others, less affected activity was completely restored, and even exceeded pre-occlusion level during the period of the occlusion. Restoration of contractile activity in the marginal zone is supposed to be due to favorable redistribution of nourishing coronary blood supply from the unoccluded vessels. The super-normal activity frequently observed during occlusion period was considered to result from the release of endogenous catecholamines induced by partial ischemia in the marginal zone following coronary occlusion. ${ }^{\mathbf{1 6},{ }^{17} \text { ) }}$

\section{ACKNOWLEDGEMENTS}

We acknowledge the skill and technical assistance of Miss Fumiko Minami. The typing and editorial efforts of Miss Yuriko Murata are also gratefully acknowledged.

\section{REFERENCES}

1. Gleason WL, Braunwald E: Studies on the first deprivative of the left ventricular pressure pulse in man. J Clin Invest 41: 80, 1962

2. Frank $O$ : On the dynamics of cardiac muscle. Am Heart J 58: 282, 1959

3. Wallace AG, Skinner NS, Jr, Mitchell JH: Hemodynamic determinants of the maximal rate of rise of left ventricular pressure. Am J Physiol 205: 30, 1963

4. Mason DT: Usefulness and limitations of the rate of rise of intraventricular pressure (dP/dt) in the evaluation of myocardial contractility in man. Am J Cardiol 23: 516, 1969

5. Cohn PF, Liedtke AJ, Serur J, Sonnenblick EH, Urschel CW: Maximal rate of pressure fall (peak negative $\mathrm{dP} / \mathrm{dt}$ ) during ventricular relaxation. Cardiovasc Res 6: 263, 1972

6. Fogelman AM, Abbasi AS, Pearce ML, Kuttus AA: Echocardiographic study of the abnormal motion of the posterior left ventricular wall during angina pectoris. Circulation 46 : 
905,1972

7. Watanabe $\mathbf{T}$, Shintani $\mathbf{F}$, Fu LT, Kato $\mathrm{K}$ : Changes in peak negative $\mathrm{LV} \mathrm{dP} / \mathrm{dt}$ following experimental coronary occlusion. The Heart 5: 1235, 1973 (in Japanese)

8. Watanabe T, Shintani F, Fu LT: Maximal rate of left ventricular pressure fall in early stage of experimental myocardial infarct. Clin Res 22: 310, 1974

9. McLaurin LP, Rolet EL, Grossman W: Impaired left ventricular relaxation during pacinginduced ischemia. Am J Cardiol 32: 751, 1973

10. Dwyer CM: Left ventricular pressure-volume alterations and regional disorders of contraction during myocardial ischemia induced by atrial pacing. Circulation 42: 1111, 1970

11. Linhart JW: Hemodynamic consequences of pacing-induced changes in heart rate in valvular aortic stenosis. Circulation 45: 300, 1972

12. Smith McK, Ratshin RA, Harrel FE, Jr, Russel RO, Jr, Rackley CE: Early sequential changes in left ventricular dimensions and filing pressure in patients after myocardial infarction. Am J Cardiol 33: 363, 1974

13. Tyberg JV, Parmley WW, Sonnenblick EH: In-vitro studies of myocardial asynchrony and regional hypoxia. Circulat Res 25: 569, 1969

14. Tyberg JV, Yeatman LA, Parmley WW, Urschel GW, Sonnenblick EH: Effects of hypoxia on mechanics of cardiac contraction. Am J Physiol 218: 1780, 1970

15. Bing OHL, Keefe JF, Wolk MJ, Finkelstein LJ, Levine HJ: Tension prolongation during recovery from myocardial hypoxia. J Clin Invest 50: 660, 1971

16. Sedek $G$, Lewartowski B: Mechanism of changes in left ventricular function after acute experimental coronary occlusion. Cardiovasc Res 7: 313, 1973

17. Barrera F, Ascanio G, Kolatat T, Tallaria RJ, Oppenheimer MJ: Interrelations of cardiac necrosis, acute hypotension and ventricular fibrillation. Am Heart J 75: 421, 1968 